\title{
A Case of Refractory Absence Epilepsy Precedes Anti-MOG Associated Optic Neuritis
}

\author{
Katharina Blunschi ${ }^{1}$, Pallavi Avasarala ${ }^{2,4}$, John Schreiber ${ }^{3,4}$, Neha Athale ${ }^{3,4}$, Ilana Kahn ${ }^{3}$, \\ Tarannum Lateef ${ }^{3,4}, *$ \\ ${ }^{1}$ Cleveland Clinic Lerner College of Medicine, Case Western Reserve University, Cleveland, OH, USA \\ ${ }^{2}$ Trinity College of Arts and Sciences, Duke University, Durham, NC, USA \\ ${ }^{3}$ Department of Neurology, Children's National Health System and George Washington University School of Medicine, Washington D. C., \\ USA \\ ${ }^{4}$ Department of Neurology, Pediatric Specialists of Virginia, Fairfax, VA, USA
}

Email address:

tlateef@childrensnational.org (T. Lateef)

*Corresponding author

\section{To cite this article:}

Katharina Blunschi, Pallavi Avasarala, John Schreiber, Neha Athale, Ilana Kahn, Tarannum Lateef. A Case of Refractory Absence Epilepsy Precedes Anti-MOG Associated Optic Neuritis. Clinical Neurology and Neuroscience. Vol. 5, No. 2, 2021, pp. 25-29. doi: $10.11648 /$ j.cnn.20210502.15

Received: April 5, 2021; Accepted: May 11, 2021; Published: May 27, 2021

\begin{abstract}
Childhood absence epilepsy (CAE) is one of the most common forms of pediatric epilepsy. While most patients become seizure free with anti-epileptic drug therapy approximately $20 \%$ do not achieve seizure remission and are defined as having refractory CAE. Epilepsy is generally thought of as a grey matter disease but has also been associated with abnormal white matter. Optic neuritis $(\mathrm{ON})$, on the other hand, is typically a white matter disorder characterized by inflammation and demyelination of the myelin sheath due to autoantibodies, such as the anti-myelin oligodendrocyte glycoprotein (MOG) antibody. We present the case of an 11-year-old female with refractory CAE who developed anti-MOG antibody positive ON. $\mathrm{CAE}$ and $\mathrm{ON}$ are not commonly co-morbid and to our knowledge their co-occurrence in a patient has not been previously described. However, in this case, the CAE and $\mathrm{ON}$ may be related as her seizures dramatically improved after initiating immunomodulatory treatments for the ON. This may indicate a relationship between ON (potentially specific to anti-MOG positive $\mathrm{ON}$ ) and $\mathrm{CAE}$, or may suggest that there is an inflammatory component to CAE and that immunomodulatory therapies may have a role in seizure control. Thus, in cases of treatment resistant absence epilepsy, an immune work up may be helpful.
\end{abstract}

Keywords: Seizure, Childhood Absence Epilepsy, Neuroinflammation, Demyelination

\section{Introduction}

Childhood absence epilepsy (CAE) is one of the most common forms of pediatric epilepsy, accounting for approximately $10 \%$ of epilepsies in children [1]. The syndrome involves absence seizures, which are characterized by sudden, frequent, short periods of altered consciousness without a loss of muscle tone. While most patients become seizure free with anti-epileptic drug therapy, such as ethosuximide, approximately $20 \%$ do not achieve seizure remission and are defined as having refractory CAE [2].

Epilepsy is generally thought of as a grey matter disease. However, it can less frequently be associated with abnormal white matter as well $[3,4]$. Optic neuritis $(\mathrm{ON})$, on the other hand, is typically a white matter disorder, and can be characterized by autoantibodies against components of the myelin sheath, such as anti- myelin oligodendrocyte glycoprotein (MOG) antibody, which result in inflammation and subsequent demyelination $[5,6]$.

We present the case of an 11-year-old female with refractory childhood absence epilepsy who developed antiMOG antibody positive $\mathrm{ON}$ and had subsequent improvement in her seizures after treatment with steroids and rituximab for $\mathrm{ON}$. CAE and $\mathrm{ON}$ are not commonly comorbid and to our knowledge their co-occurrence in a patient has not been previously described. However, in this case, the 
CAE and ON appear to be related as her seizures dramatically improved after initiating immunomodulatory treatments for the $\mathrm{ON}$.

\section{Case Presentation}

The patient is an 11-year-old Caucasian right-handed female with refractory absence epilepsy. She was initially diagnosed with classic childhood absence epilepsy at 3.5 years of age, based on clinical presentation and supporting EEG criteria, which responded well to low dose ethosuximide. She was weaned off ethosuximide at age 6 years 11 months and remained seizure free off anti-epileptic medication for one year with a normal EEG during this period. At age 8 (June 2017) she had recurrence of seizures, leading to an admission for EEG which was most consistent with generalized absence epilepsy with some focal features, namely apparent propagation of generalized epileptiform activity fairly consistently from the right frontal region (Figure 1).

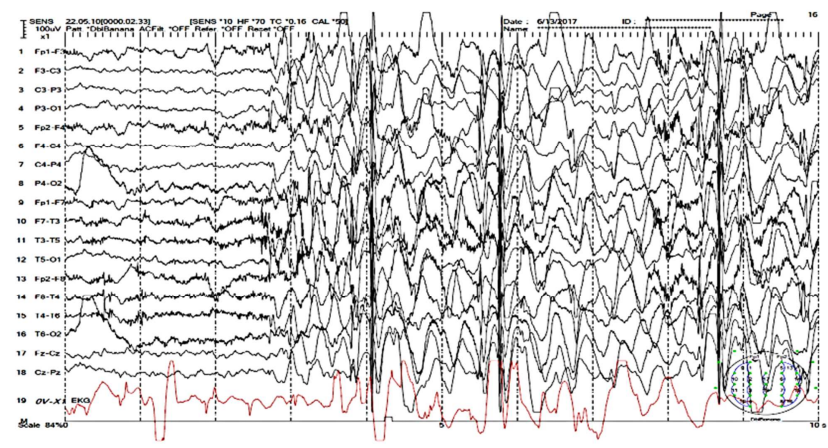

Figure 1. Segment of continuous EEG recording from June 2017. Awake EEG recording displayed in a double banana montage demonstrating a burst of irregular, generalized, anterior-predominant $3.5 \mathrm{~Hz}$ spike-wave discharges. High frequency filter $70 \mathrm{~Hz}$, time constant 0.16 seconds, calibration bar included on top left of figure, 10 seconds per page.

A non-contrast MRI of her brain was normal at the time (Figure 2).

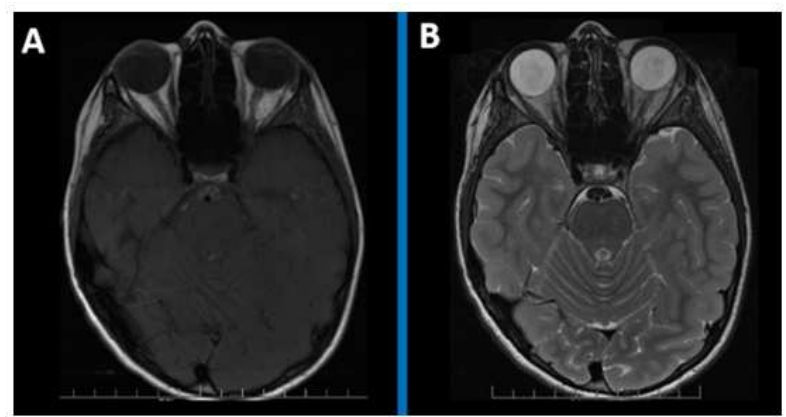

Figure 2. Normal Brain MRI from June 2017. T1 (A) and T2 (B)-weighted images consistent with normal brain MRI. This MRI was obtained after patient's seizure recurrence and prior to her optic neuritis presentation.

Ethosuximide was restarted at this time, but seizures persisted and remained long in duration (lasting a few minutes as opposed to typical CAE seizures which last seconds) despite increasing the dose of ethosuximide multiple times. Due to the patient's atypical absence epilepsy not responding to ethosuximide, the decision was made to transition to valproic acid and slowly wean ethosuximide. She was stabilized on a regimen of ethosuximide and valproic acid for a short time, remaining seizure free for four weeks. She then had a recurrence of worsening seizures with more clustering and longer seizure duration associated with enuresis resulting in another admission for seizure work-up and management during which clobazam was added. EEG during this second hospital admission remained similar to previous, notable for frequent bursts of generalized discharges at 2.5 and $3 \mathrm{~Hz}$, lasting 3-7 seconds (Figure 3).

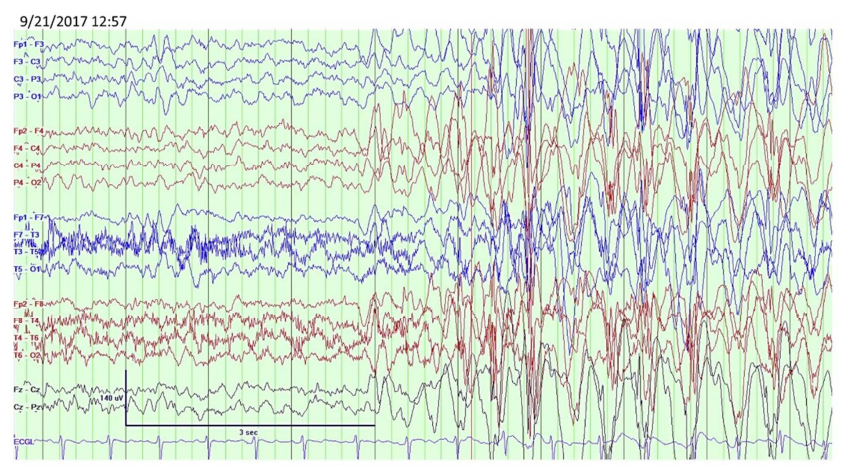

Figure 3. Segment of a continuous EEG recording from September 2017. Awake EEG recording displayed in a double banana montage demonstrating a burst of generalized, anterior-predominant $3.5 \mathrm{~Hz}$ spike-wave discharges. High frequency filter $70 \mathrm{~Hz}$, low frequency filter $1 \mathrm{~Hz}, 10$ seconds per page.

Her seizures became increasingly difficult to control despite a multi-drug AED regimen consisting of ethosuximide (low dose), valproic acid, and clobazam. She also began to experience a decline in school performance during this period, specifically in math, and experienced additional adverse effects including hair loss, tremor, cognitive issues, thrombocytopenia, leukopenia, and elevated AST. Ethosuximide was weaned off completely and the decision was made to add lamotrigine and decrease valproic acid to better manage side effects, with her regimen now consisting of clobazam $7.5 \mathrm{mg}$ twice per day, valproic acid $375 \mathrm{mg}$ twice per day, and lamotrigine $50 \mathrm{mg}$ twice per day. VEEG repeated during this time remained notable for irregular, generalized $3 \mathrm{~Hz}$ spike-wave discharges, usually more prominent or starting on the right side, particularly the right parietal-posterior temporal region (Figure 4).

These findings were interpreted as most indicative of a risk for primarily generalized seizures with focal features vs focal seizures emanating from deep cortical structures within the right central-parietal-temporal region.

Then, approximately 10 months after seizure recurrence, the patient suddenly developed right temporal headache, pain with movement of the right eye, blurry vision, and light sensitivity. Work-up at that time, including a dilated ophthalmologic exam, revealed abnormal optic nerve findings subsequently confirmed by MRI to be ON. Further testing revealed the presence of anti-MOG antibodies associated with the optic neuritis. She was treated with high 
dose pulse steroids for three days after which her vision improved and eye pain resolved. MRI of the patient's brain during her hospital admission was consistent with right optic neuritis and interval mild cerebral atrophy or pseudo atrophy (Figure 5).

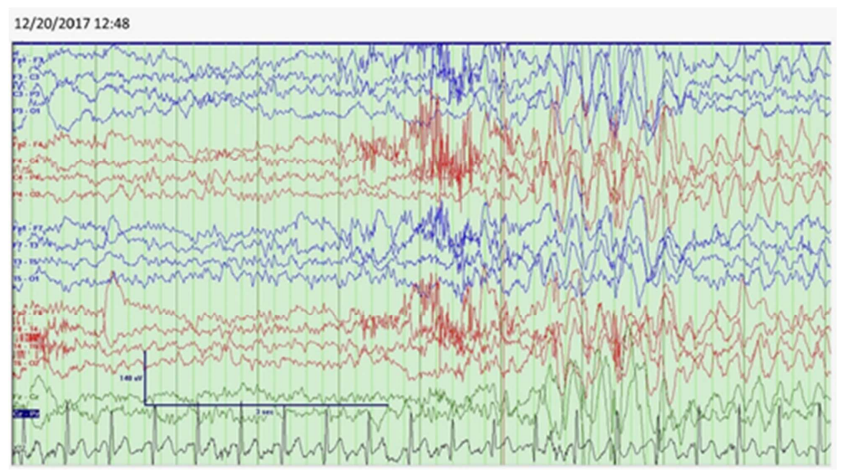

Figure 4. Segment of a continuous EEG recording from December 2017. Awake EEG recording displayed in a double banana montage demonstrating a burst of generalized, right central-parietal-temporal-predominant $3.5 \mathrm{~Hz}$ spike-wave discharges. High frequency filter $70 \mathrm{~Hz}$, low frequency filter 1 $\mathrm{Hz}, 10$ seconds per page.
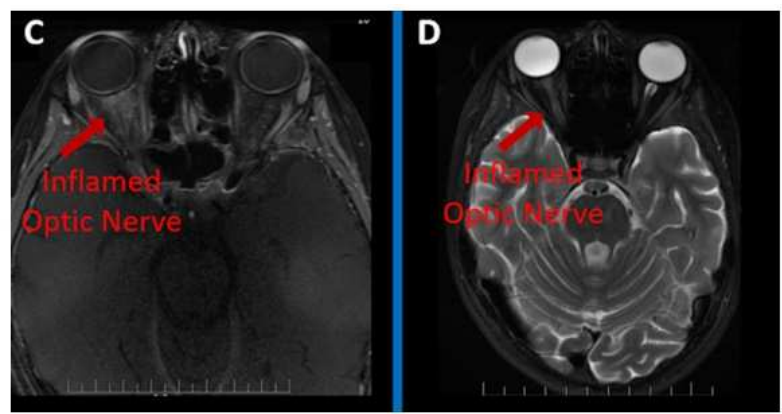

Figure 5. Brain MRI from April 2018 consistent with optic neuritis. T1 (C) and T2 (D) - weighted Brain MRI images demonstrating poor delineation of the retrobulbar soft tissue surrounding the right optic nerve sheath and enhancement of the orbital right optic nerve (see arrows) consistent with optic neuritis.

MRI spine revealed no abnormalities. After discharge she was followed up in neuroimmunology clinic where she was started on rituximab infusions, which she received in two week courses every six months.

She underwent a follow up continuous four-hour VEEG with photic stimulation and hyperventilation two months after admission, which was now normal in the awake and asleep states with no focal, paroxysmal, or epileptiform abnormalities identified (Figure 6).

Clobazam was weaned and eventually discontinued entirely after another two months (four months from admission). Follow up MRI Brain seven months after admission revealed resolution of right optic nerve inflammation and mild improvement of presumed cerebral pseudoatrophy (Figure 7).

She has now remained seizure free on a stable regimen of lamotrigine $50 \mathrm{mg}$ twice per day and valproic acid $375 \mathrm{mg}$ twice per day for 1.5 years. She continues to receive rituximab infusions every six months and has received nine infusions to date.

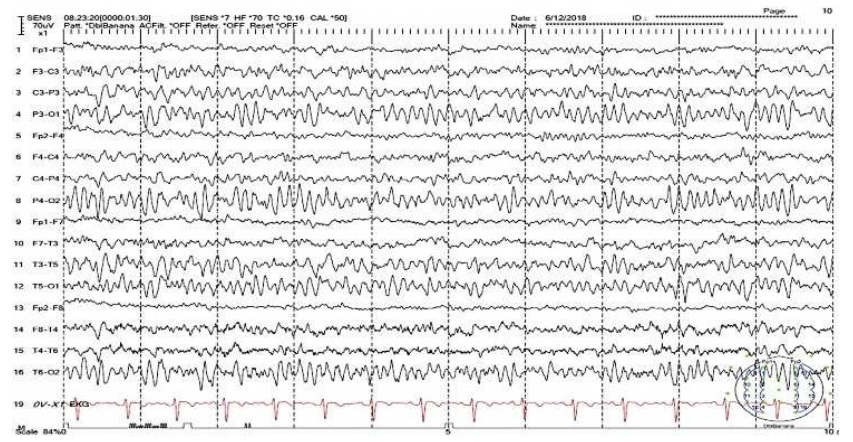

Figure 6. Segment of a continuous four-hour EEG recording from June 2018. Awake EEG recording displayed in a double banana montage demonstrating a normal awake background without epileptiform activity. High frequency filter $70 \mathrm{~Hz}$, time constant 0.16 seconds, calibration bar included on top left of figure, 10 seconds per page.
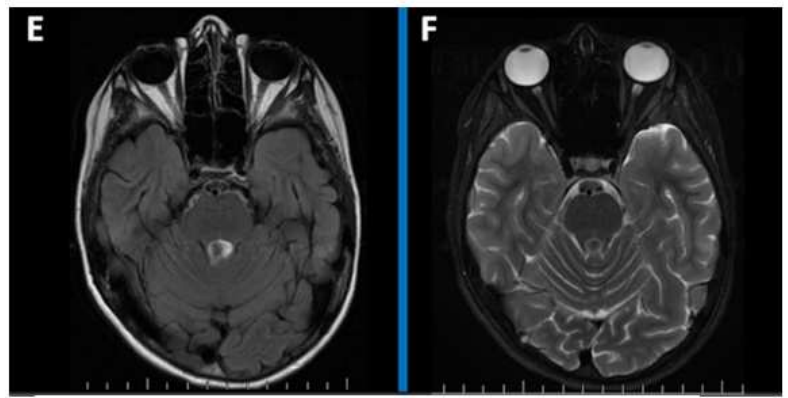

Figure 7. Normal Brain MRI from November 2018. T1 (E) and T2 (F) weighted Brain MRI images demonstrating resolution of right optic nerve inflammation seen on prior April 2018 study.

\section{Discussion}

In summary, this is an interesting case of an 11-year-old female with a history of classic CAE who, after being seizure free for one year, had recurrence of refractory seizures and then subsequent development of ON 10 months later. Her seizures were finally controlled, EEG normalized, and clobazam successfully weaned after the patient received pulse dose steroids and rituximab for ON.

This case may indicate a relationship between $O N$ (potentially specific to anti-MOG positive ON) and CAE or may suggest that there is an inflammatory component to $\mathrm{CAE}$, or seizure exacerbation in CAE, and that immunomodulatory therapies may have a role in seizure control. These possible relationships are supported by previous studies on CAE. Patients with CAE typically have normal clinical MRIs, however various microstructural abnormalities are found in both grey and white matter [7, 8]. CAE patients have been found to have smaller gray matter volumes of the left orbital frontal gyrus, bilateral temporal lobes, subcallosal gyrus, and bilateral thalami [7, 8]. They have also been found to have reduced white matter in the extranuclear subcortical area and basal forebrain, and white matter abnormalities in the basal ganglia thalamocortical circuit [7, 9]. Moreover, Liang et al. associated epileptic activity during absence seizures to microstructural changes in 
the white matter areas of the seizure discharge [3]. It is important to note that while these grey and white matter changes were found to be statistically significant, their clinical significance is not certain.

$\mathrm{ON}$ is characterized by inflammation and subsequent demyelination of the optic nerve [5]. Demyelination can occur in $\mathrm{ON}$ through autoantibodies, such as the anti-MOG antibody, which targets the myelin oligodendrocyte glycoprotein (MOG) a component of the CNS myelin sheath [6]. A few case studies have linked anti-MOG antibodies to epilepsy. According to Hamid et al., $14.7 \%$ of patients testing positive for MOG antibody had seizures [10]. Other case reports presented patients with anti-MOG positive ON who developed seizures as the disease progressed [11]. In addition, Gutman et al. reviewed eleven patients with anti-MOG positive $\mathrm{ON}$, four of whom experienced generalized seizures [12].

The association between epilepsy and anti-MOG antibodies provides further support for the hypothesis that epilepsy has an inflammatory pathology, like ON. Neuroinflammation has now increasingly been shown to play a significant role in childhood epilepsies [13]. While the brain was previously thought of as an immune privileged zone, recent studies have demonstrated that breakdown of the blood-brain-barrier has a role in seizure initiation and progression in both animal and human models, and that multiple immune cell types including $\mathrm{T}$ cells, macrophages, and dendritic cells can in fact traverse the blood-brain-barrier [13]. One study examining cortical tissue collected from 13 patients who underwent surgery for intractable epilepsy demonstrated glial activation and cell injury as well as increased levels of pro-inflammatory cytokines including IL-1 $\beta$, Il-8, IL-12p70, and MIP-1 $\beta$ in epileptogenic cortex [14]. Another study employed PET translocator protein imaging to show the presence of inflammation in neocortical epilepsy [15].

The relationship between epilepsy and neuroinflammation is further supported by the increased probability of seizures in patients with Multiple Sclerosis (MS), a T-cell mediated demyelinating autoimmune disease, with a mechanism of BBB breakdown [16]. A study by Lapato et al. found that inducing demyelination resulted in activation of inflammatory mediators and seizure onset [17]. Furthermore, a case study presenting a patient with both MS and seizures, found that treatment for MS lead to a decrease in seizures, similar to our case presentation [16]. This suggests that immune dysfunction could be an underlying cause for seizure occurrence, just as it is an underlying cause for demyelination.

This is supported by several studies that have found a decrease in seizure activity in response to anti-inflammatory and immunomodulatory drugs. Anti-inflammatory or immunomodulatory treatments such as steroids, intravenous immunoglobulin (IVIG), plasma exchanges, rituximab, and cyclophosphamide have resulted in a decrease in seizure occurrence in animal models [18]. In humans, immunomodulatory therapies have been shown to play a role in the treatment of refractory childhood epilepsy syndromes such as Doose syndrome (myoclonic-astatic epilepsy), electrical status epilepticus in sleep (ESES), and infantile spasms [19-21].

\section{Conclusion}

While epilepsy has been associated with anti-MOG diseases and response to anti-inflammatory and immunomodulatory drugs, this is the first case to our knowledge presenting a relationship between CAE and antiMOG optic neuritis specifically, where both the intractable absence seizures and the ON responded to immunotherapy. This case suggests that refractory absence epilepsy may have immunologic underpinnings and an immune work up may be helpful in guiding further treatment.

\section{Abbreviations}

CAE: Childhood Absence Epilepsy; ON: Optic Neuritis; MOG: Myelin Oligodendrocyte Glycoprotein; AED: Antiepileptic drug; EEG: Electroencephalogram; VEEG: Video Electroencephalogram; MRI: Magnetic Resonance Imaging; PET: Positron Emission Tomography; CNS: Central Nervous System; ESES: electrical status epilepticus in sleep; IVIG: intravenous immunoglobulin; mg: milligrams.

\section{References}

[1] Posner E. Absence seizures in children. BMJ Clin Evid. Jan 10 $2008 ; 2008$.

[2] Arya R, Greiner HM, Lewis A, et al. Vagus nerve stimulation for medically refractory absence epilepsy. Seizure. May 2013; 22 (4): 267-70. doi: 10.1016/j.seizure.2013.01.008.

[3] Liang JS, Lee SP, Pulli B, et al. Microstructural Changes in Absence Seizure Children: A Diffusion Tensor Magnetic Resonance Imaging Study. Pediatr Neonatol. Aug 2016; 57 (4): 318-25. doi: 10.1016/j.pedneo.2015.10.003.

[4] Widjaja E, Kis A, Go C, Raybaud C, Snead OC, Smith ML. Abnormal white matter on diffusion tensor imaging in children with new-onset seizures. Epilepsy Res. Mar 2013; 104 (1-2): 105-11. doi: 10.1016/j.eplepsyres.2012.10.007.

[5] Foroozan R, Buono LM, Savino PJ, Sergott RC. Acute demyelinating optic neuritis. Curr Opin Ophthalmol. Dec 2002; 13 (6): 375-80.

[6] Dos Passos GR, Oliveira LM, da Costa BK, et al. MOG-IgGAssociated Optic Neuritis, Encephalitis, and Myelitis: Lessons Learned From Neuromyelitis Optica Spectrum Disorder. Front Neurol. 2018; 9: 217. doi: 10.3389/fneur.2018.00217.

[7] Chan CH, Briellmann RS, Pell GS, Scheffer IE, Abbott DF, Jackson GD. Thalamic atrophy in childhood absence epilepsy. Epilepsia. Feb 2006; 47 (2): 399-405. doi: 10.1111/j.15281167.2006.00435.x.

[8] Caplan R, Levitt J, Siddarth P, et al. Frontal and temporal volumes in Childhood Absence Epilepsy. Epilepsia. Nov 2009; 50 (11): 2466-72. doi: 10.1111/j.1528-1167.2009.02198.x. 
[9] Rossi MA. Deep white matter track record of functional integrity in childhood absence epilepsy. Epilepsy Curr. Nov 2012; 12 (6): 234-5. doi: 10.5698/1535-7511-12.6.234.

[10] Hamid SHM, Whittam D, Saviour M, et al. Seizures and Encephalitis in Myelin Oligodendrocyte Glycoprotein IgG Disease vs Aquaporin $4 \mathrm{IgG}$ Disease. JAMA Neurol. Jan 1 2018; 75 (1): 65-71. doi: 10.1001/jamaneurol.2017.3196.

[11] Zhong X, Zhou Y, Chang Y, et al. Seizure and Myelin Oligodendrocyte Glycoprotein Antibody-Associated Encephalomyelitis in a Retrospective Cohort of Chinese Patients. Front Neurol. 2019; 10: 415. doi: 10.3389/fneur.2019.00415.

[12] Gutman JM, Kupersmith M, Galetta S, Kister I. Anti-myelin oligodendrocyte glycoprotein (MOG) antibodies in patients with optic neuritis and seizures. J Neurol Sci. Apr 15 2018; 387: 170-173. doi: 10.1016/j.jns.2018.01.042.

[13] Koh S. Role of Neuroinflammation in Evolution of Childhood Epilepsy. J Child Neurol. Jan 2018; 33 (1): 64-72. doi: 10.1177/0883073817739528.

[14] Choi J, Nordli DR, Jr., Alden TD, et al. Cellular injury and neuroinflammation in children with chronic intractable epilepsy. J Neuroinflammation. Dec 19 2009; 6: 38. doi: 10.1186/1742-2094-6-38.

[15] Dickstein LP, Liow JS, Austermuehle A, et al. Neuroinflammation in neocortical epilepsy measured by PET imaging of translocator protein. Epilepsia. Jun 2019; 60 (6): 1248-1254. doi: 10.1111/epi.15967.
[16] Sotgiu S, Murrighile MR, Constantin G. Treatment of refractory epilepsy with natalizumab in a patient with multiple sclerosis. Case report. BMC Neurol. Sep 23 2010; 10: 84. doi: 10.1186/1471-2377-10-84.

[17] Lapato AS, Szu J, Hasselmann JPC, Khalaj AJ, Binder DK, Tiwari-Woodruff SK. Chronic demyelination-induced seizures. Neuroscience. Jan 30 2017; 346: 409-422. doi: 10.1016/j.neuroscience.2017.01.035.

[18] Korff CM, Dale RC. The Immune System in Pediatric Seizures and Epilepsies. Pediatrics. Sep 2017; 140 (3) doi: 10.1542/peds.2016-3534.

[19] Kelley SA, Kossoff EH. Doose syndrome (myoclonic-astatic epilepsy): 40 years of progress. Dev Med Child Neurol. Nov 2010 ; 52 (11): 988-93. doi: 10.1111/j.14698749.2010.03744.x.

[20] Veggiotti P, Pera MC, Teutonico F, Brazzo D, Balottin U, Tassinari CA. Therapy of encephalopathy with status epilepticus during sleep (ESES/CSWS syndrome): an update. Epileptic Disord. Mar 2012; 14 (1): 1-11. doi: 10.1684/epd.2012.0482.

[21] Song JM, Hahn J, Kim SH, Chang MJ. Efficacy of Treatments for Infantile Spasms: A Systematic Review. Clin Neuropharmacol. Mar/Apr 2017; 40 (2): 63-84. doi: 10.1097/WNF.00000000000002. 